\title{
Light Scattering and Sedimentation Equilibrium Study on Concentrated Solutions of a Rodlike Polysaccharide Schizophyllan
}

\author{
Kazuo VAN and Akio Teramoto* \\ Department of Macromolecular Science, Osaka University, \\ Toyonaka, Osaka 560, Japan
}

(Received September 11, 1984)

\begin{abstract}
Light scattering and sedimentation equilibrium measurements were made on concentrated solutions of a rodlike polysaccharide schizophyllan using three samples of different molecular weights. The data were analyzed to obtain the partial derivative $\left[\partial \Delta \mu_{0} / \partial \phi\right]_{M n}$ of the excess chemical potential of the solvent with respect to the polymer volume fraction $\phi$. The results for isotropic solutions were analyzed by the theory of Flory and Abe for rodlike polymers, yielding a non-vanishing interaction parameter $\chi$. For any of the samples investigated, $\chi$ was negative and decreased with increasing $\phi$. No appreciable dependence of $\chi$ on molecular weight was found. Sedimentation measurement was also made on biphasic mixtures consisting of isotropic and cholesteric phases at equilibrium to evaluate $\left[\partial \Delta \mu_{0} / \partial \phi\right]_{\mathrm{Mn}}$ at the phase boundary in the two phases. No theory was found to describe precisely our experimental data.

KEY WORDS Chemical Potential / Rodlike Polymer / Polysaccharide / Light Scattering / Sedimentation Equilibrium / Schizophyllan / Interaction Parameter / Flory Theory / Onsager Theory / Thermodynamics /
\end{abstract}

A solution of a rodlike polymer forms an anisotropic phase at high concentration, and separates into equilibrium isotropic and anisotropic phases at a certain intermediate concentration. Such phase behavior as well as the thermodynamic properties of these phases can in principle be predicted if relevant expressions are available for the chemical potentials of the components involved. The theories so far developed along this line of thought indicate that an asymmetric shape of the polymer is of primary importance for the formation of an anisotropic phase. ${ }^{1-4}$ However, it is predict$\mathrm{ed}^{1-3}$ that intermolecular interactions also play an important role in the phase behavior. Thus, knowledge of the chemical potentials is essential for testing theoretically predicted phase relationships. As far as we are aware, attempts to approach such knowledge have been made only by Kubo and Ogino ${ }^{5,6}$ who studied the system poly( $\gamma$-benzyl L-glutamate) + dimethylformamide. They obtained thermodynamic data that could be compared favorably with the prediction of Cotter's theory. ${ }^{4}$ Applicability of this theory to other systems is yet to be seen.

For the reason given below, aqueous schizophyllan, in which this rodlike polysaccharide $^{7,8}$ forms a cholesteric mesophase at high concentrations, ${ }^{9-11}$ cannot be regarded as an athermal solution of hard rods in a noninteracting medium. The average value of the second virial coefficient for aqueous schizophyllan at $25^{\circ} \mathrm{C}$ is $1.25 \times 10^{-4} \mathrm{~mol} \mathrm{~cm}^{3} \mathrm{~g}^{-2}{ }^{7,8}$ Analysis of this value by Zimm's theory of rodlike polymers ${ }^{12}$ gives $1.2 \mathrm{~nm}$ for the diam-

* To whom correspondence should be addressed. 
eter of the schizophyllan triple helix. This is smaller than $1.67 \mathrm{~nm}$ calculated from the partial specific volume of schizophyllan in water at infinite dilution $\left(0.619 \mathrm{~cm}^{3} \mathrm{~g}^{-1}\right)$ and the molecular weight per unit length $M_{\mathrm{L}}$ of 2140 $\mathrm{nm}^{-1}$ and also smaller than the hydrodynamic diameter $2.2-2.6 \mathrm{~nm}$ reported by Norisuye and coworkers. ${ }^{7,8}$ These disparities suggest that concentrated aqueous schizophyllan is non-athermal and its thermodynamic properties cannot be described in terms of the geometry of the schizophyllan helix alone. Therefore, we attempted to directly evaluate the thermodynamic properties of aqueous schizophyllan. Thus, light scattering and sedimentation equilibrium measurements were made on isotropic solutions and sedimentation measurement was made on biphasic mixtures. The resulting data were treated according to Scholte $^{13,14}$ to obtain the solvent chemical potential. This paper describes the experimental results and their comparison with theoretical predictions.

\section{EXPERIMENTAL}

\section{Polysaccharide Samples}

Sonicated schizophyllan samples supplied by Taito Co. were purified or separated into many fractions from aqueous solutions with methanol or acetone as the precipitant in our laboratory. Five fractions were chosen from them and designated as SPG-3' $\left(M_{w}=159000\right.$, $\left.M_{z} / M_{w}=1.2\right)$, R23 $\left(M_{w}=245000, M_{z} / M_{w}=\right.$ 1.2), D40 $\left(M_{w}=478000, M_{z} / M_{w}=1.2\right)$, SPG$01\left(M_{v}=105000\right)$, and E-4 $\left(M_{v}=150000\right)$. The molecular weights of these samples were determined by sedimentation equilibrium measurement or viscosity measurement on dilute solutions at $25^{\circ} \mathrm{C}$; the intrinsic viscosity $[\eta]-$ $M_{w}$ relationship of Yanaki et $a .^{8}{ }^{8}$ was used to obtain the viscosity-average molecular weight $M_{v}$. For light scattering measurement, sample R23 was dissolved in water at about $1 \mathrm{wt} \%$, filtered through a Millipore filter GSWP0 2500 of pore size $0.22 \mu \mathrm{m}$, and freeze-dried.
A required amount of a schizophyllan sample was dried in vacuo overnight and mixed with water in a stoppered flask. The mixture became transparent within two days. Dissolution was effected by slowly rotating the flask or by stirring the mixture by a magnetic bar. The polymer weight fraction $w$ was determined gravimetrically except for R23 solutions; those of R23 solutions were determined by refractometry after the light scattering measurement.

\section{Refractive Index and Density Measurements}

Refractive indices $n$ for light of the wavelengths 436 and $546 \mathrm{~nm}$ were measured on aqueous solutions of the sample E-4 over the polymer concentration range up to $15 \mathrm{wt} \%$ at $25^{\circ} \mathrm{C}$. A Bausch \& Lomb precision sugar refractometer was used. The values of $n$ obtained are plotted against $w$ in Figure 1, where the solid curves follow the data points for the respective wavelengths. Owing to the difficulty in handling highly viscous solutions and a limited amount of the sample available, no sufficient data were obtained to determine the $n$ vs. $w$ relationship accurately. Therefore, we searched for appropriate relationships giving an initial slope consistent with the literature specific refractive index increment at infinite

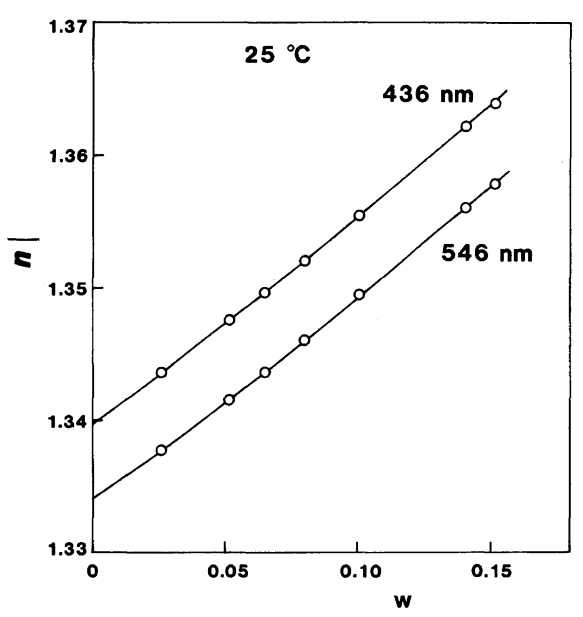

Figure 1. Concentration dependence of refractive index of aqueous schizophyllan at $25^{\circ} \mathrm{C}$; solid lines, calculated from eq 1 . 
dilution $^{7}$ and a reasonable $n$ value at $w=1$, i.e., for the pure polymer. The resulting equations are

$$
\begin{gathered}
n=1.3339+0.1416 w+0.123 w^{2}-0.03124 w^{3} \\
(546 \mathrm{~nm}) \\
n=1.3397+0.1446 w+0.115 w^{2}-0.0243 w^{3} \\
(436 \mathrm{~nm})
\end{gathered}
$$

Densities of aqueous solutions of sample SPG-01 were measured on a Lipkin-Davison type pycnometer of $5 \mathrm{~cm}^{3}$ capacity over the concentration range up to $9.5 \mathrm{wt} \%$ at $25^{\circ} \mathrm{C}$. The data for the specific volume $v$ of isotropic solutions obtained were fitted by the equation:

$$
v=1.003-0.384 w+0.03 w^{2}
$$

This was determined by the same method as used in deriving eq 1 .

\section{Light Scattering Measurement}

A FICA 50 automatic photogoniometer was used with cylindrical cells of $1.8 \mathrm{~cm}$ in diameter. Intensities of scattered light were measured for vertically polarized incident light of the wavelengths 546 and $436 \mathrm{~nm}$ at scattering angles between 30 and $150^{\circ}$. Appropriate neutral density filters were used to attenuate strong $436 \mathrm{~nm}$ intensities. The transmittances of the filters were determined from the ratio of the $90^{\circ}$ scattering intensities measured with and without the filter on aqueous schizophyllan or a polystyrene-xylene solution. The photogoniometer was calibrated with benzene according to the procedure established in our laboratory. Test solutions were filtered through a Millipore filter of the pore size $0.22 \mu \mathrm{m}$ and centrifuged directly in the light scattering cell.

\section{Sedimentation Equilibrium Measurement}

Sedimentation equilibrium measurement on isotropic solutions was made by using a Beckman-Spinco Model E analytical ultracentrifuge equipped with a schlieren optical system at $25^{\circ} \mathrm{C}$. A Kel-F $12 \mathrm{~mm}$ cell or an
Epon $30 \mathrm{~mm}$ double-sector cell was used and the solution column was adjusted to a length between 0.7 and $1.5 \mathrm{~mm}$. The rotor speed was chosen at $3000-4000 \mathrm{rpm}$.

For a biphasic mixture, an appropriate volume of it was placed in a Kel-F cell and the whole cell assembly was kept at $30^{\circ} \mathrm{C}$ at least for two days to effect phase separation. The cell assembly was then loaded on a J-rotor heated at $30^{\circ} \mathrm{C}$ and centrifuged at a rotor speed between 2000 and $5600 \mathrm{rpm}$. The length of the solution column was adjusted between 1.5 and $4.3 \mathrm{~mm}$.

\section{TREATMENT OF LIGHT SCATTERING AND SEDIMENTATION EQUILIBRIUM DATA}

Consider a solution consisting of a polymer and a single solvent; the polymer may or may not be monodisperse in molecular weight. In a light scattering measurement, vertically polarized light is incident on the solution and the intensity of scattered light is measured as a function of the scattering angle $\theta$ to obtain the reduced excess scattering intensity $R_{\theta}$. The quantity $Z_{\mathrm{LS}}$ defined by

$$
Z_{\mathrm{LS}}=-\frac{R T K M_{0} w v}{R_{0}}
$$

can be related to the thermodynamic properties of the solution. Here, $R_{0}$ is the value of $R_{\theta}$ extrapolated to the zero scattering angle, $M_{0}$ the molecular weight of the solvent, ${ }^{15} v$ the specific volume of the solution, and $K$ the optical constant defined by

$$
K=\frac{4 \pi^{2} n^{2}(\partial n / \partial w)^{2}}{N_{\mathrm{A}} \lambda_{0}^{4}}
$$

with $N_{\mathrm{A}}$ the Avogadro number, $\partial n / \partial w$ the specific refractive index increment on the weight fraction basis, and $\lambda_{0}$ the wavelength of incident light in vacuo. For a monodisperse solute, $Z_{\mathrm{LS}}$ is equal to the partial derivative $\left[\partial \Delta \mu_{0} / \partial w\right]$ of the solvent chemical potential with respect to $w$ at fixed temperature and 
pressure. ${ }^{13,14}$

For aqueous schizophyllan, the values of $\partial n / \partial w$ and $v$ as functions of $w$ can be obtained using the relations established (eq 1 and 2). Thus, with the known values of $N_{\mathrm{A}}$ and $\lambda_{0}$, light scattering measurement allows $Z_{\mathrm{LS}}$ to be evaluated for isotropic solutions of schizophyllan. In so doing, $M_{0}$ was taken to be 2200 for the reason given in the following section.

Next, consider the same polymer solution at sedimentation equilibrium in an ultracentrifuge cell. A sedimentation equilibrium measurement gives the refractive index gradient $\mathrm{d} n / \mathrm{d} r$ in the solution as a function of the distance $r$ from the center of rotation. A quantity $Z_{\text {SED }}$ (essentially the same as $Z_{\mathrm{LS}}$ ) is obtained from $\mathrm{d} n / \mathrm{d} r$ by

$$
Z_{\mathrm{SED}}=\frac{M_{0} w(r) r \omega^{2}(\partial v / \partial w)(\partial n / \partial w)}{v(\mathrm{~d} n / \mathrm{d} r)}
$$

where $\omega$ is the angular velocity, $w(r)$ the polymer weight fraction at $r$, and $\partial v / \partial w$ the partial derivative of $v$ with respect to $w$. When the rotor speed is low and the solution column is short, the concentration gradient developed in the cell is almost constant throughout the entire solution and $w(r)$ at the midpoint of the solution is very close to the weight fraction in the original solution, which is hereafter denoted by $w$. Thus, the sedimentation equilibrium method allows us to evaluate $Z_{\text {SED }}$ for a known $w$. A similar analysis may be made for phase-separated solutions as well if $w(r)$ at the phase boundary can be determined.

Actually, sedimentation equilibrium measurements on isotropic solutions of schizophyllan were carried out under the conditions specified above and the data were analyzed by eq 5 along with the data for $\partial n / \partial w, v$, and $\partial v / \partial w$ presented in the Experimental section. Similar measurements were also made on biphasic mixtures, using solution columns somewhat longer than those for the isotropic solutions.

In what follows, we express $Z_{X}(X=\mathrm{LS}$, SED) in terms of the polymer volume fraction $\phi$ defined by

$$
\phi=w v_{\mathrm{p}} /\left[w v_{\mathrm{p}}+(1-w) v_{0}\right]
$$

where $v_{\mathrm{p}}$ is the partial specific volume of the polymer at infinite dilution and $v_{0}$ the specific volume of the pure solvent; $v_{\mathrm{p}}=0.619 \mathrm{~cm}^{3} \mathrm{~g}^{-1}$, and $v_{0}=1.0029 \mathrm{~cm}^{3} \mathrm{~g}^{-1}$. Thus, we use the quantities $\left[\partial \Delta \mu_{0} / \partial \phi\right]_{X}$ defined by

$\left[\partial \Delta \mu_{0} / \partial \phi\right]_{X}=Z_{X}(\partial w / \partial \phi) \quad(X=\mathrm{LS}, \mathrm{SED})$

\section{EXPERIMENTAL RESULTS}

\section{Light Scattering Data}

Figure 2 shows the scattering angle $\theta$ dependence of $n^{2} / R_{\theta}$ for light of wavelength $546 \mathrm{~nm}$ at different polymer concentrations. It can be seen that the data points at each concentration are fitted closely by a straight line, allowing an accurate estimation of the ordinate intercept $n^{2} / R_{0}$ to be made, where $n$ is

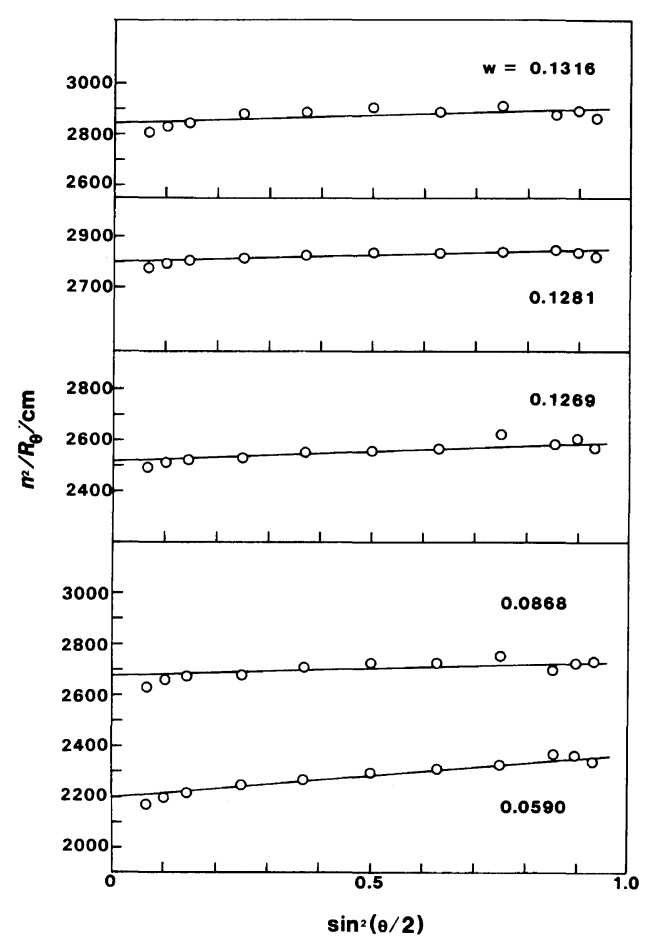

Figure 2. Plots of $n^{2} / R_{\theta}$ for light of wavelength $546 \mathrm{~nm}$ vs. $\sin ^{2}(\theta / 2)$ for isotropic solutions of sample $\mathrm{R} 23$ at $25^{\circ} \mathrm{C}$. 
Table I. Light scattering data for aqueous solutions of $\mathrm{R} 23$ at $25^{\circ} \mathrm{C}$

\begin{tabular}{|c|c|c|c|c|c|c|c|c|c|}
\hline \multirow{3}{*}{$w$} & \multirow{3}{*}{$\phi$} & \multicolumn{4}{|c|}{$546 \mathrm{~nm}$} & \multicolumn{4}{|c|}{$436 \mathrm{~nm}$} \\
\hline & & $n^{2} / R_{0}$ & $\left\langle S^{2}\right\rangle_{\mathrm{app}}^{1 / 2}$ & $-\left[\partial \Delta \mu_{\mathrm{o}} / \partial \phi\right]_{\mathrm{LS}}$ & $-\left[\partial \Delta \mu_{\mathrm{o}} / \partial \phi\right]_{\mathrm{Mn}}$ & $n^{2} / R_{0}$ & $\left\langle S^{2}\right\rangle_{\mathrm{app}}^{1 / 2}$ & $-\left[\partial \Delta \mu_{\mathrm{o}} / \partial \phi\right]_{\mathrm{LS}}$ & $-\left[\partial \Delta \mu_{\mathrm{o}} / \partial \phi\right]_{\mathrm{Mn}}$ \\
\hline & & $\mathrm{cm}$ & $\mathrm{nm}$ & $\mathrm{J} \mathrm{mol}^{-1}$ & $\mathrm{~J} \mathrm{~mol}^{-1}$ & $\mathrm{~cm}$ & $\mathrm{~nm}$ & $\mathrm{~J} \mathrm{~mol}^{-1}$ & $\mathrm{~J} \mathrm{~mol}^{-1}$ \\
\hline 0.0590 & 0.0373 & 2190 & 34 & 198 & 207 & 900 & 36 & 200 & 209 \\
\hline 0.0868 & 0.0554 & 2670 & 25 & 362 & 370 & 1075 & 26 & 365 & 373 \\
\hline 0.1269 & 0.0823 & 2510 & 34 & 531 & 539 & 1010 & 38 & 532 & 540 \\
\hline 0.1281 & 0.0831 & 2800 & 29 & 597 & 605 & 1080 & 32 & 575 & 583 \\
\hline 0.1316 & 0.0855 & 2480 & 33 & 627 & 635 & 1120 & 26 & 616 & 624 \\
\hline
\end{tabular}

the refractive index of the solution. The resulting values of $R_{0}$ are used to calculate $Z_{\mathrm{LS}}$ by the procedure described in the previous section. The data at $436 \mathrm{~nm}$ showed substantially the same trend as those at $546 \mathrm{~nm}$ and analyzed in the same way. The numerical data of $Z_{\mathrm{LS}}$ for sample R23 are summarized in Table I. The values of $Z_{\mathrm{LS}}$ at 546 and $436 \mathrm{~nm}$ agree with each other, except for the case of $w=0.1281$.

From the slope $S_{0}$ of the straight line in Figure 2 we calculated the quantity $\left\langle S^{2}\right\rangle_{\text {app }}$ defined by

$$
\left\langle S^{2}\right\rangle_{\text {app }}=3 c \lambda_{0}^{2} M_{w} K_{\mathrm{c}} S_{0} /\left(16 \pi^{2} n^{2}\right)
$$

where $c$ is the mass concentration of the polymer and $K_{\mathrm{c}}$ is equal to $K$ with $\partial n / \partial w$ replaced by $\partial n / \partial c .\left\langle S^{2}\right\rangle_{\text {app }}$ reduces to the mean-square radius of gyration of the polymer, $\left\langle S^{2}\right\rangle_{z}$ at infinite dilution. It can be seen in Table I that the values of $\left\langle S^{2}\right\rangle_{\text {app }}$ are close to $33 \mathrm{~nm}$, which is the $\left\langle S^{2}\right\rangle_{z}$ value interpolated at the sample's $M_{w}$ from the $\left\langle S^{2}\right\rangle_{z}-M_{w}$ relationship of Kashiwagi et al. ${ }^{16}$ This agreement may be taken to guarantee the accuracy of $R_{\theta}$. Indeed, our experience showed that $\left\langle S^{2}\right\rangle_{\text {app }}$ was larger for larger $R_{\theta}$, indicating that optical purification was not satisfactory in such a case.

\section{Sedimentation Equilibrium Data for Isotropic Solutions}

Sedimentation equilibrium measurements on isotropic solutions of samples R23, D40, and SPG-3' were performed using relatively short solution columns and low rotor speeds, so that centrifugal fractionation might be minimized. The results obtained at different rotor speeds agreed with each other within the experimental error. The results are given in Tables II through IV.

\section{Chemical Potential of the Solvent}

According to Flory and Abe, ${ }^{17}$ the chemical potential of the solvent in an isotropic solution of polydisperse rods is expressed as

$$
\Delta \mu_{0}=R T\left[\ln (1-\phi)+\left(1-1 / x_{n}\right) \phi+\chi \phi^{2}\right]
$$

where $x_{n}$ is the number-average axial ratio and $\chi$ is the interaction parameter which may depend on $\phi: \chi=0$ for an athermal solution. Except for some trivial terms, this expression and those for the solute chemical potentials are the same as their counterparts for isotropic solutions of flexible polymers. Therefore, we use Scholte's methods ${ }^{13,14}$ for flexible polymers to obtain the solvent chemical potential from light scattering and sedimentation equilibrium data.

His method utilizes the relation:

$$
\begin{aligned}
{\left[\partial \Delta \mu_{0} / \partial \phi\right]_{\mathrm{Mn}}=} & {\left[\partial \Delta \mu_{0} / \partial \phi\right]_{X} } \\
- & R T M_{0}\left(1 / M_{n}-1 / M_{w}\right)(\partial w / \partial \phi) \\
& (X=\mathrm{LS}, \mathrm{SED})
\end{aligned}
$$

where the subscript $\mathrm{Mn}$ indicates that the differentiation is performed at fixed $M_{n}$. This relation shows that both light scattering and sedimentation equilibrium measurements should give the same information, i.e., $Z_{\mathrm{LS}}=$ 
Table II. Sedimentation equilibrium data for concentrated isotropic solutions of sample $\mathrm{R} 23$ at $25^{\circ} \mathrm{C}$

\begin{tabular}{|c|c|c|c|c|c|}
\hline \multirow{2}{*}{$w$} & \multirow{2}{*}{$\phi$} & Rotor speed & \multirow{2}{*}{$\frac{\mathrm{d} n / \mathrm{d} r}{10^{-3} \mathrm{~cm}^{-1}}$} & \multirow{2}{*}{$\frac{-\left[\partial \Delta \mu_{\mathrm{o}} / \partial \phi\right]_{\mathrm{SED}}}{\mathrm{J} \mathrm{mol}^{-1}}$} & \multirow{2}{*}{$\frac{-\left[\partial \Delta \mu_{\mathrm{o}} / \partial \phi\right]_{\mathrm{Mn}}}{\mathrm{J} \mathrm{mol}^{-1}}$} \\
\hline & & $\mathrm{rpm}$ & & & \\
\hline 0.059 & 0.0373 & 4400 & 8.69 & 210 & 219 \\
\hline 0.0868 & 0.0554 & & 8.44 & 331 & 339 \\
\hline 0.1281 & 0.0831 & & 8.09 & 527 & 535 \\
\hline
\end{tabular}

Table III. Sedimentation equilibrium data for isotropic solutions of sample D40 at $25^{\circ} \mathrm{C}$

\begin{tabular}{|c|c|c|c|c|c|}
\hline \multirow{2}{*}{$w$} & \multirow{2}{*}{$\phi$} & Rotor speed & \multirow{2}{*}{$\frac{\mathrm{d} n / \mathrm{d} r}{10^{-3} \mathrm{~cm}^{-1}}$} & \multirow{2}{*}{$\frac{-\left[\partial \Delta \mu_{\mathrm{o}} / \partial \phi\right]_{\mathrm{SED}}}{\mathrm{J} \mathrm{mol}^{-1}}$} & \multirow{2}{*}{$\frac{-\left[\partial \Delta \mu_{\mathrm{o}} / \partial \phi\right]_{\mathrm{Mn}}}{\mathrm{J} \mathrm{mol}^{-1}}$} \\
\hline & & $\mathrm{rpm}$ & & & \\
\hline 0.0196 & 0.0122 & 2980 & 3.88 & 69.8 & 74.4 \\
\hline 0.0321 & 0.0200 & & 4.13 & 108 & 113 \\
\hline \multirow[t]{2}{*}{0.0494} & 0.0311 & 2980 & 4.28 & 165 & 169 \\
\hline & & 3370 & 5.42 & 163 & 167 \\
\hline 0.0618 & 0.0391 & 2980 & 4.11 & 216 & 220 \\
\hline 0.0712 & 0.0452 & & 4.14 & 251 & 255 \\
\hline 0.0794 & 0.0505 & & 4.07 & 286 & 290 \\
\hline 0.0905 & 0.0579 & & 4.10 & 327 & 331 \\
\hline
\end{tabular}

Table IV. Sedimentation equilibrium data for isotropic solutions of sample SPG- $3^{\prime}$ at $25^{\circ} \mathrm{C}$

\begin{tabular}{|c|c|c|c|c|c|}
\hline \multirow{2}{*}{$w$} & \multirow{2}{*}{$\phi$} & \multirow{2}{*}{$\begin{array}{c}\text { Rotor speed } \\
\text { rpm }\end{array}$} & \multirow{2}{*}{$\frac{\mathrm{d} n / \mathrm{d} r}{10^{-3} \mathrm{~cm}^{-1}}$} & \multirow{2}{*}{$\frac{-\left[\partial \Delta \mu_{\mathrm{o}} / \partial \phi\right]_{\mathrm{SED}}}{\mathbf{J} \mathrm{mol}^{-1}}$} & \multirow{2}{*}{$\frac{-\left[\partial \Delta \mu_{\mathrm{o}} / \partial \phi\right]_{\mathrm{Mn}}}{\mathrm{J} \mathrm{mol}^{-1}}$} \\
\hline & & & & & \\
\hline 0.0246 & 0.0153 & 3370 & 3.43 & 129 & 143 \\
\hline 0.0249 & 0.0155 & & 3.65 & 122 & 136 \\
\hline 0.0503 & 0.0316 & & 4.21 & 218 & 231 \\
\hline 0.0700 & 0.0444 & & 4.29 & 303 & 316 \\
\hline 0.0745 & 0.0473 & & 4.36 & 318 & 331 \\
\hline 0.0981 & 0.0629 & & 4.19 & 445 & 458 \\
\hline 0.1082 & 0.0697 & & 4.07 & 509 & 522 \\
\hline 0.1285 & 0.0834 & & 3.82 & 654 & 667 \\
\hline 0.1539 & 0.1009 & & 3.80 & 801 & 813 \\
\hline
\end{tabular}

$Z_{\mathrm{SED}}$.

In our analysis, $M_{w} / M_{n}$ was assumed to be 1.25 for all the samples. ${ }^{18}$ The schizophyllan triple helix was approximated by a uniform cylinder of the diameter $d$, and the axial ratio $x_{n}$ was calculated from $M_{n}$ by

$$
x_{n}=M_{r} v_{\mathrm{p}} /\left(N_{\mathrm{A}} \pi d^{3} / 4\right)
$$

Here, as mentioned in the Introduction, $d$ was taken to be $1.67 \mathrm{~nm}$ to be consistent with the helix geometry. ${ }^{7,8}$ The molecular weight $M_{0}$ of the solvent was calculated from the relation: $M_{0}=\left(N_{\mathrm{A}} \pi d^{3} / 4\right) / v_{0}$ to be 2210 . The values obtained for $\left[\partial \Delta \mu_{0} / \partial \phi\right]_{\mathrm{Mn}}$ are summarized in Tables II through IV. As seen in these tables, the polydispersity correction expressed by the 


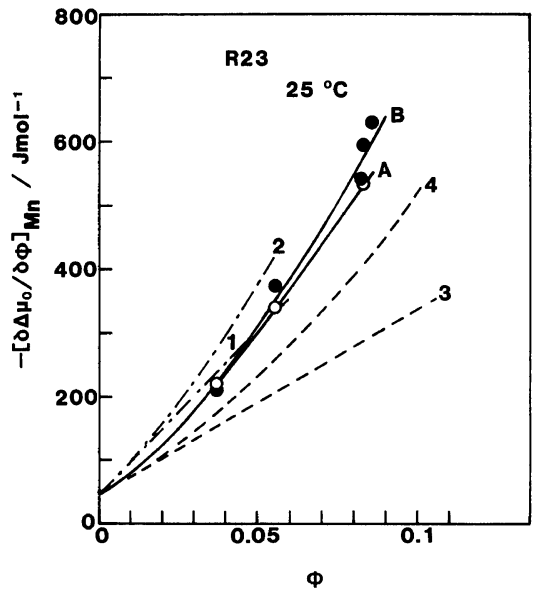

Figure 3. Plot of $-\left[\partial \Delta \mu_{0} / \partial \phi\right]_{\mathrm{Mn}} v s$. $\phi$ for isotropic solutions of sample R23 at $25^{\circ} \mathrm{C}$. Unfilled circles, sedimentation equilibrium data; filled circles, light scattering data. Solid curve B, calculated from the Flory-Abe theory (eq 9) with eq 17 and $x_{n}=54.7$; solid curve A, with eq 16 and $x_{n}=54.7$. Dot-dash curves 1 and 2, calculated for $x=54.7$ from the Onsager and Cotter theories, respectively; dashed curves 3 and 4, for $x=29.3$ (see the text). Each of the theoretical curves $1-4$ is terminated at $\phi_{\mathrm{A}}$, the polymer volume fraction at the isotropicbiphasic boundary.

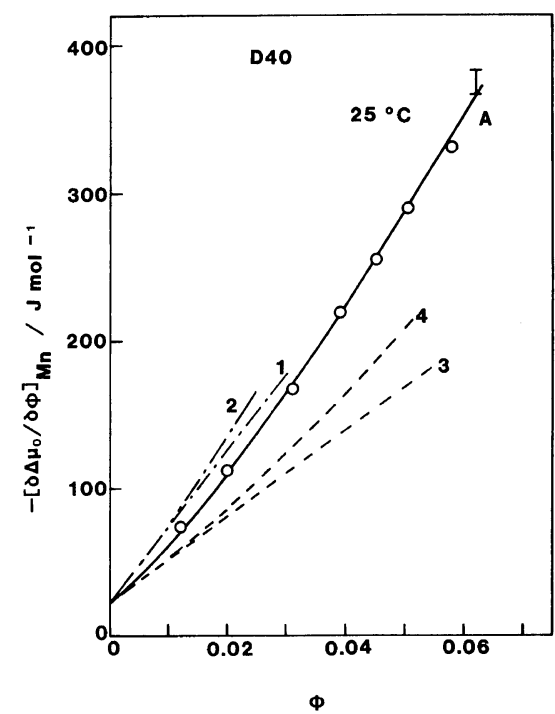

Figure 4. Dependence of $-\left[\partial \Delta \mu_{0} / \partial \phi\right]_{\mathrm{Mn}}$ on $\phi$ for sample $\mathrm{D} 40$ at $25^{\circ} \mathrm{C}$. Solid curve A, calculated by eq 9 with eq 14 and $x_{n}=107$; dot-dash curves 1 and 2, calculated for $x=107$ from the Onsager and Cotter theories; dashed curves 3 and 4 , for $x=59.4$ (see the text).

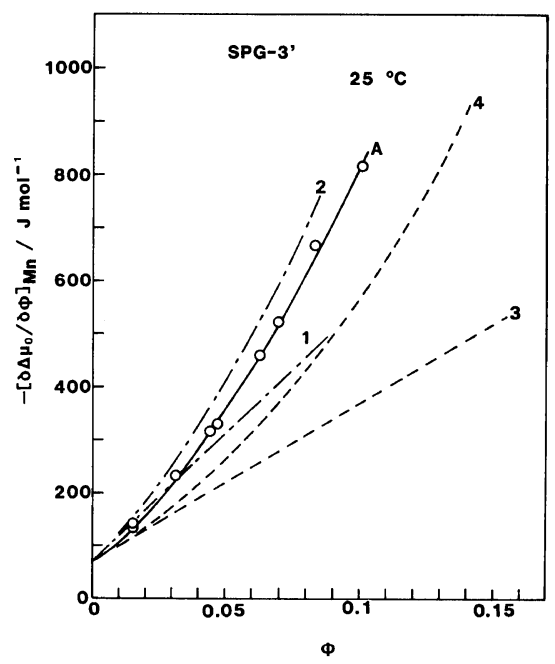

Figure 5. Dependence of $-\left[\partial \Delta \mu_{0} / \partial \phi\right]_{\mathrm{Mn}}$ on $\phi$ for sample SPG- $3^{\prime}$ at $25^{\circ} \mathrm{C}$. Solid curve A, calculated by eq 9 with eq 15 and $x_{n}=35.5$; dot-dash curves 1 and 2, calculated for $x=35.5$ from the Onsager and Cotter theories; dashed curves 3 and 4, for $x=19.2$ (see the text).

second term on the right-hand side of eq 10 , which is not very appreciable even at small $\phi$, becomes rapidly negligible with increasing $\phi$.

Figure 3 shows the values of $\left[\partial \Delta \mu_{0} / \partial \phi\right]_{M n}$ for sample R23 as a function of $\phi$. The light scattering (filled circles) and sedimentation equilibrium (unfilled circles) data are consistent with each other but, not exactly. The discrepancy is more conspicuous at higher concentrations, but the reason is not clear. Figures 4 and 5 show plots of $\left[\partial \Delta \mu_{0} / \partial \phi\right]_{\mathrm{Mn}}$ for the samples D40 and SPG-3'.

\section{DISCUSSION}

\section{Interaction Parameter}

As mentioned in the Introduction, concentrated aqueous schizophyllan may be characterized by a non-vanishing interaction parameter $\chi$ in the framework of the Flory theory. For simplicity, we assume that $\chi(\phi)$ is expanded by a polynomial in $\phi$ as

$$
\chi(\phi)=\chi_{0}+\chi_{1} \phi+\chi_{2} \phi^{2}+\chi_{3} \phi^{3}
$$

where $\chi_{i}(i=0-3)$ are coefficients dependent 
on temperature. As is well known, $\chi_{0}$ is related to the sedimentation second virial coefficient $A_{2}$ of the solution by

$$
A_{2}=\left(v_{\mathrm{p}}^{2} / M_{0} v_{0}\right)\left(1 / 2-\chi_{0}\right)
$$

Substitution of eq 12 into eq 9 followed by differentiation with respect to $\phi$ gives an explicit expression in $\phi$ for $\left[\partial \Delta \mu_{0} / \partial \phi\right]_{\mathrm{Mn}}$. Fitting experimental data for $\left[\partial \Delta \mu_{0} / \partial \phi\right]_{\mathrm{Mn}}$ to this expression allows the $\chi_{i}$ coefficients to be evaluated. This was done for the three sets of sedimentation equilibrium data, and the following results were obtained:

$$
\begin{gathered}
\chi(\phi)=-0.107-9.33 \phi+70.0 \phi^{2}-204 \phi^{3} \\
(\mathrm{D} 40) \\
\chi(\phi)=-0.055-11.41 \phi+66.9 \phi^{2}-228 \phi^{3} \\
\left(\mathrm{SPG}-3^{\prime}\right) \\
\chi(\phi)=-0.021-9.03 \phi+42.7 \phi^{2}-55 \phi^{3}
\end{gathered}
$$

where $\chi_{0}$ in each equation has been determined from available experimental data for $A_{2}$ by eq 13. A similar analysis was performed on the light scattering data for R23, yielding

$$
\chi(\phi)=-0.021-9.16 \phi+38 \phi^{2}-75.9 \phi^{3}
$$

Figure 6 shows $\chi$ plotted against $\phi$. For any sample, $\chi(\phi)$ is negative and decreases monotonously with increasing $\phi$. For sample R23,

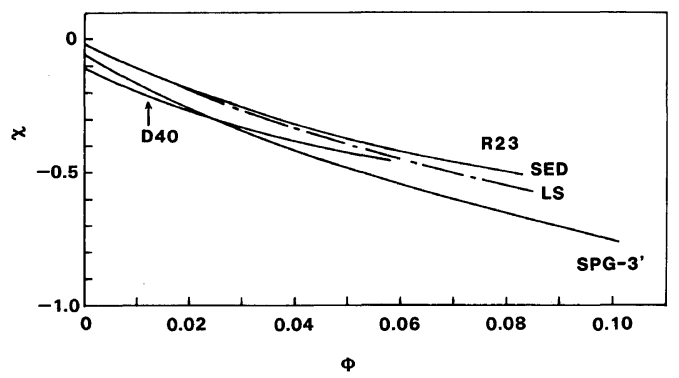

Figure 6. Plots of $\chi(\phi) v s . \phi$ for aqueous schizophyllan at $25^{\circ} \mathrm{C}$. Solid curves, determined from the sedimentation equilibrium data; dot-dash curve, from the light scattering data. the values of $\chi(\phi)$ obtained from the two different methods are in good agreement. Differences in $\chi$ among the three samples are not appreciable, where comparison can be made. The thick curves (A) in Figures 3 through 5 represent $\left[\partial \Delta \mu_{0} / \partial \phi\right]_{\mathrm{Mn}}$ calculated with the $\chi(\phi)$ given by eq $14-16$. They fit the experimental data closely. It is concluded from these findings that the system schizophyllan + water is not athermal in the framework of the FloryAbe theory.

\section{Comparison with Other Theories}

The theory of Onsager ${ }^{1}$ is concerned with an imperfect gas of cylindrical particles. It should be valid for long cylinders in dilute solution, but its validity at higher concentration may be questioned, since it relies on a free energy expression linear in $\phi$. Note that no such limitation exists in the Flory theory. ${ }^{3,17}$ Various attempts have been made to extend the Onsager theory to higher concentration. ${ }^{19-21}$ Recently, Cotter ${ }^{4}$ has modified the Onsager theory using the scaled particle theory ${ }^{22}$ and Lasher's calculation for the phase boundary concentrations. ${ }^{19} \mathrm{We}$ here confine ourselves to the Onsager theory with no intermolecular interaction and the Cotter theory in analyzing experimental data.

According to these theories, the excess chemical potential $\Delta \mu_{0}$ of the solvent is expressed in terms of the axial ratio $x$, the volume fraction $\phi$, and the molar volume $V_{\mathrm{p}}$ of the polymer as follows:

$$
\Delta \mu_{0}=-\frac{R T M_{0} v_{0}}{V_{\mathrm{p}}} f(x, \phi)
$$

Different $f(x, \phi)$ are used by different authors. The theories of Onsager and Cotter give the same expression for the second virial coefficient: ${ }^{23}$

$$
A_{2}=\left(\frac{3 x^{2}+6 x-1}{3 x-1}\right)\left(\frac{v_{\mathrm{p}}^{2}}{V_{\mathrm{p}}}\right)
$$

To a first approximation, we used the number averages of $V_{\mathrm{p}}$ and $x\left(x_{n}\right)$ to compute $\Delta \mu_{0}$ 
given by eq $18 ; x_{n}$ was calculated by eq 11 . In Figures 3 through 5 our schizophyllan data are compared with the predictions of the two theories under consideration; the theoretical curves indicated by the dot-dash lines (curves 1 and 2) are terminated at the respective Apoint. The linear dependence predicted by the Onsager theory is not in agreement with the experimental data. On the other hand, the values calculated by the Cotter theory are rather close to the experimental data for sample SPG-3'. The theoretical values tend to deviate progressively upward from the data as the molecular weight is increased. Use of the corresponding weight averages ${ }^{23}$ for analyzing $\left[\partial \Delta \mu_{0} / \partial \phi\right]_{X}(X=\mathrm{LS}, \mathrm{SED})$ led to a similar conclusion.

Kubo and Ogino ${ }^{5,6}$ showed that their osmotic pressure data for the system $\operatorname{poly}(\gamma$ benzyl L-glutamate) + dimethylformamide agreed with the prediction of the Cotter theory, when $x$ was calculated from $A_{2}$ by eq 19 . The dashed curves ( 3 and 4 ) in Figures 3-5 represent the theoretical values calculated with $x$ obtained in the same way. In contrast with the Kubo and Ogino case, no satisfactory agreement between theory and experiment is seen.

Finally, we used $x_{w}$ for $x$ in eq 18, which is obtained by $x_{w}=M_{w} /\left(d M_{\mathrm{L}}\right)$ and $d$ taken to be $2.6 \mathrm{~nm}$, the hydrodynamic diameter of the schizophyllan triple helix. ${ }^{7,8}$ The values of $\left[\partial \Delta \mu_{0} / \partial \phi\right]_{\mathrm{Mn}}$ computed by the Cotter theory ${ }^{4}$ with $x$ thus obtained are shown in Figure 7. The agreement between theory and experiment is excellent. However, it must be noted that the experimental $w_{\mathrm{A}}$ for D40 is 1.7 times as large as the theoretical $w_{\mathrm{A}}$. This discrepancy cannot be attributed to chain flexibility, against the argument of Kubo an Ogino,,6 since the triple helix in this range of molecular weight is completely rigid. ${ }^{7,8}$ Thus, the above agreement between calculated and experimental values of $\left[\partial \Delta \mu_{0} / \partial \phi\right]_{\mathrm{Mn}}$ does not necessarily support the validity of the Cotter theory. A similar discrepancy in the phase boundary concentration

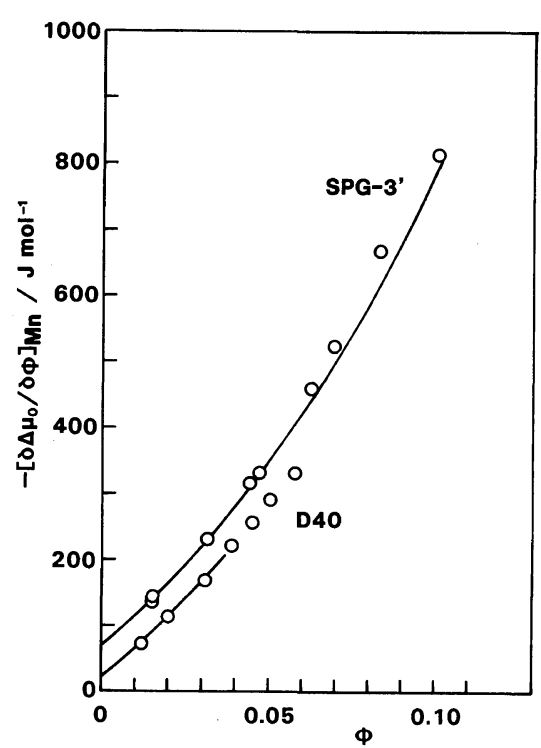

Figure 7. Dependence of $-\left[\partial \Delta \mu_{0} / \partial \phi\right]_{\mathrm{Mn}}$ on $\phi$ for isotropic solutions of samples D40 and SPB-3' at $25^{\circ} \mathrm{C}$. Solid curves, calculated from the modified Cotter theory with $x=85.4$ for D40 and 28.5 for SPG-3'.

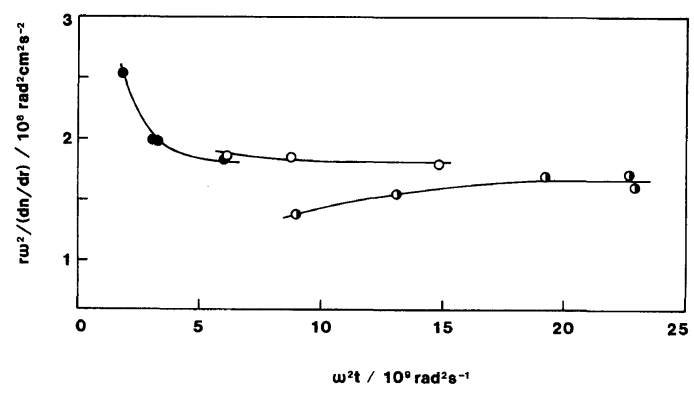

Figure 8. Plots of $r \omega^{2} /(\mathrm{d} n / \mathrm{d} r)$ vs. $\omega^{2} t$ for isotropic phases in biphasic mixtures with different overall concentration $\phi^{0}$. Unfilled circles, $\phi^{0}=0.0678$, the rotor speed $=2980 \mathrm{rpm}$; filled circles, $\phi^{0}=0.0719,1980 \mathrm{rpm}$; half-filled circles, $\phi^{0}=0.0805,2200 \mathrm{rpm}$.

is seen also in the analysis of Kubo and Ogino. In conclusion, neither Onsager theory nor Cotter theory can explain our data satisfactorily.

\section{Chemical Potential in the Biphasic Region}

When centrifuged at a rotor speed above $2000 \mathrm{rpm}$, an aqueous solution of sample D40 in the biphasic region separated into two lay- 
Table V. Sedimentation equilibrium data for biphasic solutions of sample D40 at $30^{\circ} \mathrm{C}$

\begin{tabular}{|c|c|c|c|c|c|c|}
\hline \multirow{3}{*}{$w^{\mathrm{o}}$} & \multirow{3}{*}{$\phi^{\circ}$} & \multirow{3}{*}{$\frac{\text { Rotor speed }}{\mathrm{rpm}}$} & \multicolumn{2}{|c|}{ Isotropic phase } & \multicolumn{2}{|c|}{ Cholesteric phase } \\
\hline & & & \multirow{2}{*}{$\frac{\omega^{2} r /(\mathrm{d} n / \mathrm{d} r)}{10^{8} \mathrm{rad}^{2} \mathrm{~cm}^{2} \mathrm{~s}^{-2}}$} & \multirow{2}{*}{$\frac{-\left[\partial \Delta \mu_{\mathrm{o}} / \partial \phi\right]_{\mathrm{SED}}}{\mathrm{J} \mathrm{mol}^{-1}}$} & \multirow{2}{*}{$\frac{\omega^{2} r /(\mathrm{d} n / \mathrm{d} r)}{10^{8} \mathrm{rad}^{2} \mathrm{~cm}^{2} \mathrm{~s}^{-2}}$} & \multirow{2}{*}{$\frac{-\left[\partial \Delta \mu_{\mathrm{o}} / \partial \phi\right]_{\text {SED }}}{\mathrm{J} \mathrm{mol}^{-1}}$} \\
\hline & & & & & & \\
\hline 0.1001 & 0.0644 & 2200 & 1.79 & 372 & - & - \\
\hline \multirow{2}{*}{0.1055} & 0.0678 & 2980 & 1.79 & 371 & - & - \\
\hline & & 5560 & 1.81 & 376 & - & - \\
\hline \multirow[t]{3}{*}{0.1115} & 0.0719 & 1980 & 1.81 & 376 & - & - \\
\hline & & 2980 & 1.84 & 382 & - & - \\
\hline & & 5560 & 1.79 & 371 & - & - \\
\hline 0.1192 & 0.0771 & 2200 & - & - & $0.92_{0}$ & 262 \\
\hline 0.1242 & 0.0805 & 2200 & 1.68 & 349 & $0.86_{0}$ & 245 \\
\hline
\end{tabular}

ers, isotropic and cholesteric phases. Observation by a schlieren optical system revealed that the isotropic phase appeared on top of the cholesteric phase with a wide meniscus between the two phases. The volume ratio of the two phases changed very gradually with time, suggesting that it would take quite a long time to attain a true equilibrium state. It was always possible to observe a schlieren pattern in the isotropic phase, and $\mathrm{d} n / \mathrm{d} r$ could be determined as a function of $r$. The values of $\mathrm{d} n / \mathrm{d} r$ were extrapolated to the meniscus between the two phases, $r_{\mathrm{m}}$. Figure 8 shows $r \omega^{2} /(\mathrm{d} n / \mathrm{d} r)$ at $r_{\mathrm{m}}$ plotted against $\omega^{2} t$ at different overall concentrations $\phi^{0}$, where $t$ is the time elapsed after the start of centrifugation. It can be seen that within a relatively short period of time $r \omega^{2} /$ $(\mathrm{d} n / \mathrm{d} r)$ reaches an equilibrium value which does not depend appreciably on $\phi^{0}$. From these equilibrium values of $\omega^{2} /(\mathrm{d} n / \mathrm{d} r), Z_{\mathrm{SED}}$ at $r_{\mathrm{m}}$ were calculated on the assumption that $w\left(r_{\mathrm{m}}\right)$ equals the A-point concentration at $30^{\circ} \mathrm{C}$, i.e., $w\left(r_{\mathrm{m}}\right)=0.0965$. $^{11}$ The numerical results obtained are summarized in Table V. Note that they satisfy the requirement that $Z_{\text {SED }}$ should be independent of the rotor speed.

It was possible to observe schlieren patterns of the cholesteric phase for two solutions of higher concentrations. Values of $Z_{\text {SED }}$ at the meniscus in the cholesteric phase were deter-

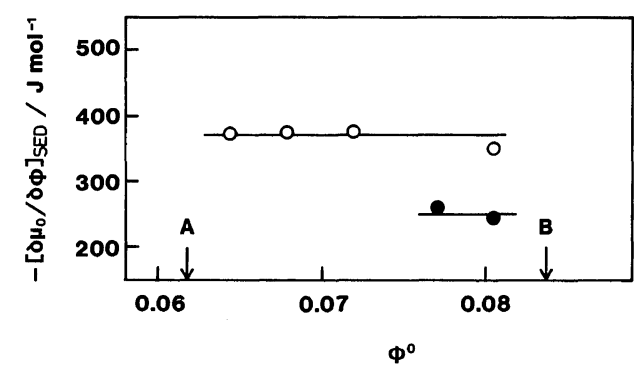

Figure 9. Plots of $-\left[\partial \Delta \mu_{0} / \partial \phi\right]_{\mathrm{SED}} v s$. $\phi^{0}$ for biphasic mixtures at $30^{\circ} \mathrm{C}$. Unfilled circles, isotropic phases; filled circles, cholesteric phases.

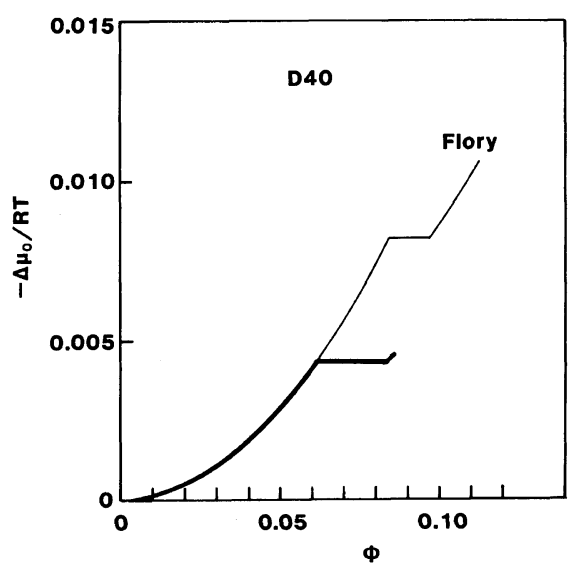

Figure 10. Excess chemical potential $\Delta \mu_{0}$ of the solvent as a function of $\phi$ for sample D40. Thick solid curve, experiment; thin curve, the Flory theory. ${ }^{3}$ 
mined by assuming that $w\left(r_{\mathrm{m}}\right)$ in the cholesteric phase was equal to the B-point concentration, 0.129. ${ }^{11}$ The numerical results are also given in Table $\mathrm{V}$.

Figure 9 shows $\left[\partial \Delta \mu_{0} / \partial \phi\right]_{\mathrm{SED}}$ at the meniscus plotted against $\phi^{0}$, where the arrows A and B indicate the A- and B-points, respectively. It can be seen that, in either phase, $\left[\partial \Delta \mu_{0} / \partial \phi\right]_{\mathrm{SED}}$ is almost constant; $\left[\partial \Delta \mu_{0} / \partial \phi\right]_{\mathrm{SED}}=-375 \mathrm{~J} \mathrm{~mol}^{-1}$ in the isotropic phase and $-250 \mathrm{~J} \mathrm{~mol}^{-1}$ in the cholesteric phase. If it is assumed that the chemical potential of the solvent does not depend appreciably on temperature, these data may be compared with those for isotropic solutions at $30^{\circ} \mathrm{C}$. Figure 4 shows that the latter can be smoothly extrapolated to the meniscus value (vertical segment).

Integration of $\left[\partial \Delta \mu_{0} / \partial \phi\right]_{\mathrm{Mn}}$ by $\phi$ along with the meniscus value and eq 14 provides $\Delta \mu_{0}$ as a function of $\phi$; the result is shown in Figure 10. The thin curve represents the Flory theory with the non-vanishing $\chi$ given by eq 14 . The agreement between theory and experiment is not satisfactory because of the disagreement in phase boundary concentration.

\section{REFERENCES}

1. L. Onsager, Ann. N. Y. Acad. Sci., 51, 627 (1947).

2. A. Ishihara, J. Chem. Phys., 19, 1142 (1951).

3. P. J. Flory, Proc. R. Soc., London, Ser. A, 234, 73 (1956).

4. M. A. Cotter, Phys. Rev. A., 10, 629 (1974).
5. K. Kubo and K. Ogino, Mol. Cryst. Liq. Cryst., 53, 207 (1979).

6. K. Kubo, Mol. Cryst. Liq. Cryst., 74, 71 (1981).

7. T. Norisuye, T. Yanaki, and H. Fujita, J. Polym. Sci., Polym. Phys. Ed., 18, 547 (1980).

8. T. Yanaki, T. Norisuye, and H. Fujita, Macromolecules, 13, 1462 (1980).

9. K. Van, T. Norisuye, and A. Teramoto, Mol. Cryst. Liq. Cryst., 78, 123 (1981).

10. K. Van and A. Teramoto, Polym. J., 14, 999 (1982).

11. T. Itou, K. Van, and A. Teramoto, J. Appl. Polym. Sci., in press.

12. B. H. Zimm, J. Chem. Phys., 14, 164 (1946).

13. Th. G. Scholte, J. Polym. Sci., A-2, 8, 841 (1970).

14. Th. G. Scholte, Eur. Polym. J., 6, 1063 (1970).

15. $Z_{X}(X=\mathrm{LS}, \mathrm{SED})$ refers to a given amount of the solvent. Therefore, $M_{\mathrm{o}}$ is the molecular weight of the "equivalent solvent" corresponding to this amount and does not necessarily equal that of water.

16. Y. Kashiwagi, T. Norisuye, and H. Fujita, Macromolecules, 14, 1220 (1981).

17. P. J. Flory and A. Abe, Macromolecules, 11, 1119 (1978).

18. This value of $M_{w} / M_{n}$ was obtained from the observed $M_{z} / M_{w}$ by assuming that the molecular weight distribution of each sample was of the Schulz type.

19. G. Lasher, J. Chem. Phys., 53, 4141 (1970).

20. J. P. Straley, Mol. Cryst. Liq. Cryst., 22, 333 (1973).

21. M. A. Cotter, J. Chem. Phys., 66, 1098 (1977).

22. H. Reiss, H. L. Frisch, and J. L. Lebowitz, J. Chem. Phys., 31, 369 (1959).

23. It can be shown on the basis of the Onsager theory that for a polydisperse sample, the light scattering and sedimentation equilibrium second virial coefficients are related to $M_{w}$, whereas the osmotic one to $M_{n}$. However, there is no method for evaluating the polydispersity effect on $\left[\partial \Delta \mu_{\mathrm{o}} / \partial \phi\right]_{X}(X=\mathrm{LS}, \mathrm{SED})$ at high concentration. Thus, it is a reasonable first approximation to use the same type of average for $V_{\mathrm{p}}$ and $x$ in eq 18 to compute theoretical values of $\Delta \mu_{\mathrm{o}}$. 